\title{
STABLE-HOMOTOPY AND HOMOLOGY INVARIANTS OF BOUNDARY LINKS
}

\author{
MICHAEL FARBER
}

\begin{abstract}
An $n$-dimensional $(n \geq 5)$ link in the $(n+2)$-dimensional sphere is stable if the $i$ th homotopy group of its complement $X$ vanishes for $2 \leq$ $i \leq(n+1) / 3$ and $\pi_{1}(X)$ is freely generated by meridians. In this paper a classification of stable links in terms of stable homotopy theory is given. For simple links this classification gives a complete algebraic description.

We also study Poincare duality in the space of the free covering of the complement of a boundary link. The explicit computation of the corresponding Ext-functors gives a construction of new homology pairings, generalizing the Blanchfield and the torsion pairings for knots.
\end{abstract}

\section{INTRODUCTION}

The present paper studies new stable-homotopy and homology invariants of boundary links of codimension two. The stable-homotopy invariant, which is called stable isometry structure, is a generalization of the corresponding notion for knots (i.e., links with one component) [F2, F3], which in its turn generalizes the Seifert matrix [L1] and the isometry structure [K] of a knot. It is shown here that one may use the Spanier-Whitehead duality theory and Wall's thickening technique [W] to obtain a complete stable-homotopy reduction of the classification problem for stable links (cf. §1.4). This reduction gives a general computational scheme which could serve as a basis of applying homotopy theory for algebraic description of different classes of stable links (as was demonstrated in [F3, F4] for stable knots).

The easiest case, where such computations can be conducted without difficulties caused by homotopy theory, is the case of simple odd-dimensional links. This class of links has been classified by Liang [L] (cf. also [Kol]) in terms of their Seifert matrices. In $\S 4$ of this paper another algebraic description of this same class of links is obtained, which is slightly better in some respects; for instance, our equivalence relation is of a more algebraic-invariant nature. Of course, the best possible form in which one could expect to get an algebraic classification of simple odd-dimensional links is the form of the Trotter-Kearton theorem [Tr, K1], which is valid for knots. Presumably a similar theorem is true

Received by the editors October 8, 1990.

1980 Mathematics Subject Classification (1985 Revision). Primary 57C45; Secondary 55D15.

Key words and phrases. Link of codimension two, Seifert surface, Spanier-Whitehead duality.

This research was supported by grant No. 88-00114 from the United States-Israel Binational Science Foundation (BSF), Jerusalem, Israel. Part of this work was written while the author was an SERC Visiting Fellow at the University of Durham. 
also for links ${ }^{1}$ and in this paper we make a step in this direction: we construct invariantly-defined Hermitian pairings on the homology of the free covering corresponding to the link, which in the case of knots represent the Blanchfield form [B] and the torsion (or linking) form [L4, F1]. Another (different) version of the Blanchfield form for boundary links was constructed by Duval [D].

\section{Preliminaries}

1.1. An $n$-dimensional $\mu$-component link is an oriented smooth submanifold $\Sigma^{n}$ of $S^{n+2}$, where $\Sigma^{n}=\Sigma_{1}^{n} \cup \cdots \cup \Sigma_{\mu}^{n}$ is the ordered disjoint union of $\mu$ submanifolds of $S^{n+2}$, each homeomorphic to $S^{n} . \Sigma$ is a boundary link if there is an oriented smooth submanifold $V^{n+1}$ of $S^{n+2}$, where $V^{n+1}=V_{1}^{n+1} \cup$ $\cdots \cup V_{\mu}^{n+1}$ is the disjoint union of connected submanifolds $V_{i}^{n+1}$, such that $\partial V_{i}=\Sigma_{i}(i=1, \ldots, \mu)$. If each $V_{i}$ is $r$-connected, we say that $V$ is an r-simple Seifert manifold for $\Sigma$.

1.2. Let $\Sigma^{n}$ be a $\mu$-component link in $S^{n+2}$, and let $X=S^{n+2}-\Sigma$ be its complement. Fix a base point $* \in X$; for each $i=1, \ldots, \mu$ the meridian $m_{i} \in \pi_{1}(X, *)$ is defined up to conjugation.

A splitting [CS] is a homomorphism (which is defined up to conjugation) $\mathscr{S}: \pi_{1}(X, *) \rightarrow F_{\mu}$ onto the free group with $\mu$ generators $t_{1}, \ldots, t_{\mu}$ that has the following property: the image of the conjugacy class $\left[m_{i}\right]$ of $i$ th meridian coincides with the conjugacy class $\left[t_{i}\right]$ of $t_{i} \in F_{\mu}$.

This notion does not depend on the choice of the base point.

Each Seifert manifold $V$ of a boundary link $\Sigma$ defines an obvious splitting $\mathscr{S}_{V}$ : if $\alpha$ is a loop in $X$ which is in general position with respect to $V$, then $\mathscr{S}_{V}([\alpha])$ is a word in $t_{1}, \ldots, t_{\mu}$, obtained by writing down $t_{i}^{\varepsilon_{i}}, \varepsilon_{i}= \pm 1$, for each intersection point $p$ of $\alpha$ and $V$ (where $i$ is the number such that $p \in V_{i} \cap \alpha$ and $\varepsilon_{i}$ is the local intersection number of $\alpha$ and $V_{i}$ at $p$ ), and then multiplying these words in the order of their appearance in $\alpha$.

A theorem of Gutiérrez [G] states that any link admitting a splitting is a boundary link (cf. also [Sm]).

1.3. An $\mathscr{F}$-link [CS] (of dimension $n$ and multiplicity $\mu$ ) is a pair $(\Sigma, \mathscr{S})$, where $\Sigma$ is a link (of dimension $n$ and multiplicity $\mu$ ) and $\mathscr{S}$ is a splitting for $\Sigma$. Two $\mathscr{F}$-links $\left(\Sigma_{1}, \mathscr{S}_{1}\right)$ and $\left(\Sigma_{2}, \mathscr{S}_{2}\right)$ are equivalent if there exists a diffeomorphism $h: S^{n+2} \rightarrow S^{n+2}$, taking $\Sigma_{1}$ onto $\Sigma_{2}$, preserving orientations of $S^{n+2}$ and $\Sigma_{\nu}, \nu=1,2$, and mapping $\mathscr{S}_{2}$ onto $\mathscr{S}_{1}$.

1.4. An $\mathscr{F}$-link $(\Sigma, \mathscr{S})$ will be called $r$-simple (where $r$ is an integer, $r \geq 1$ ) if (a) $\mathscr{S}$ is an isomorphism $\pi_{1}\left(S^{n+2}-\Sigma, *\right) \rightarrow F_{\mu}$; and (b) $\pi_{i}\left(S^{n+2}-\Sigma\right)=0$ for all $1<i \leq r$. We will consider every $\mathscr{F}$-link to be 0 -simple.

Another theorem of Gutiérrez [G] states that any $r$-simple $n$-dimensional $\mathscr{F}$ link $(\Sigma, \mathscr{S})$ admits an $r$-simple Seifert manifold $V$ with $\mathscr{S}=\mathscr{S}_{V}$, provided $n \geq 4$.

An $n$-dimensional $r$-simple $\mathscr{F}$-link will be called stable if $3 r \geq n+1 \geq 6$.

1.5. Let $V$ and $W$ be two Seifert manifolds of a boundary link $\Sigma^{n}$ in $S^{n+2}$.

\footnotetext{
${ }^{1}$ Added in proof (September 1990). The goal of obtaining an algebraic classification of simple odd-dimensional links in terms of the generalized Blanchfield pairing has been accomplished in [F5].
} 


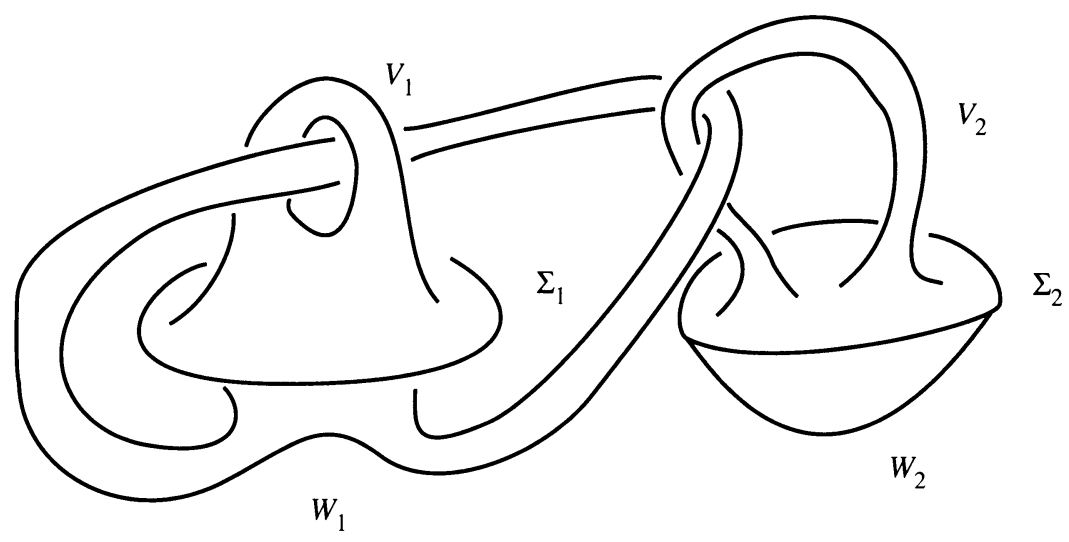

FIGURE 1

We will say that $V$ is contiguous to $W$ if

(a) $V_{i} \cap W_{j}=\varnothing$ for $i \neq j, i, j=1, \ldots, \mu$;

(b) $V_{i} \cap W_{i}=\Sigma_{i}, i=1, \ldots, \mu$;

(c) there exist $N_{i}$, the arcwise connected component of $S^{n+2}-\left(V_{i} \cup W_{i}\right)$ with the property that the positive normal to $W_{i}$ goes inside $N_{i}$, such that $\Sigma_{j} \cap N_{i}=\varnothing$ for $i, j \in\{1, \ldots, \mu\}$.

Here $\Sigma=\Sigma_{1} \cup \cdots \cup \Sigma_{\mu}, V=V_{1} \cup \cdots \cup V_{\mu}$, and $W=W_{1} \cup \cdots \cup W_{\mu}$. Of course, we assume that $V$ and $W$ are oriented and induce the same orientation on $\Sigma$.

Figure 1 shows a typical example of a pair of contiguous Seifert manifolds $V$ and $W$ of a 2-component link.

It is clear that if $V$ is contiguous to $W$ then $-W$ is contiguous to $-V$, where the minus sign means that the orientation is reversed.

Contiguous Seifert manifolds $V$ and $W$ define the same splitting: $\mathscr{S}_{V}=$ $\mathscr{S}_{W}$. The contrary is also partially true:

1.6. Theorem. Let $V$ and $W$ be two Seifert manifolds of an $n$-dimensional link $\Sigma$. If $\mathscr{S}_{V}=\mathscr{S}_{W}$ then there exists a sequence $U_{1}, \ldots, U_{N}$ of Seifert manifolds of $\Sigma$ with

(a) $U_{1}=V, U_{N}=W$;

(b) for each $i=1, \ldots, N-1$ one of the following three possibilities holds: (1) $U_{i}$ is contiguous to $U_{i+1}$; (2) $U_{i+1}$ is contiguous to $U_{i}$; (3) there is an orientation-preserving diffeomorphism $f: S^{n+2} \rightarrow S^{n+2}$ with $f\left(U_{i}\right)=$ $U_{i+1}$.

Proof. Choose a base point $* \in S^{n+2}-\Sigma$. As explained in $\S 1.2 \mathrm{~V}$ and $W$ define epimorphisms

$$
f_{V}, f_{W}: \pi_{1}\left(S^{n+2}-\Sigma\right) \rightarrow F_{\mu}
$$

and the condition $\mathscr{S}_{V}=\mathscr{S}_{W}$ means that

$$
f_{V}(x)=a f_{W}(x) a^{-1}
$$

for some element $a \in F_{\mu}$. As the first step we will describe modifications of $W$ by a chain of contiguities, which give another Seifert manifold $W^{\prime}$ with $f_{V}=f_{W^{\prime}}$. 

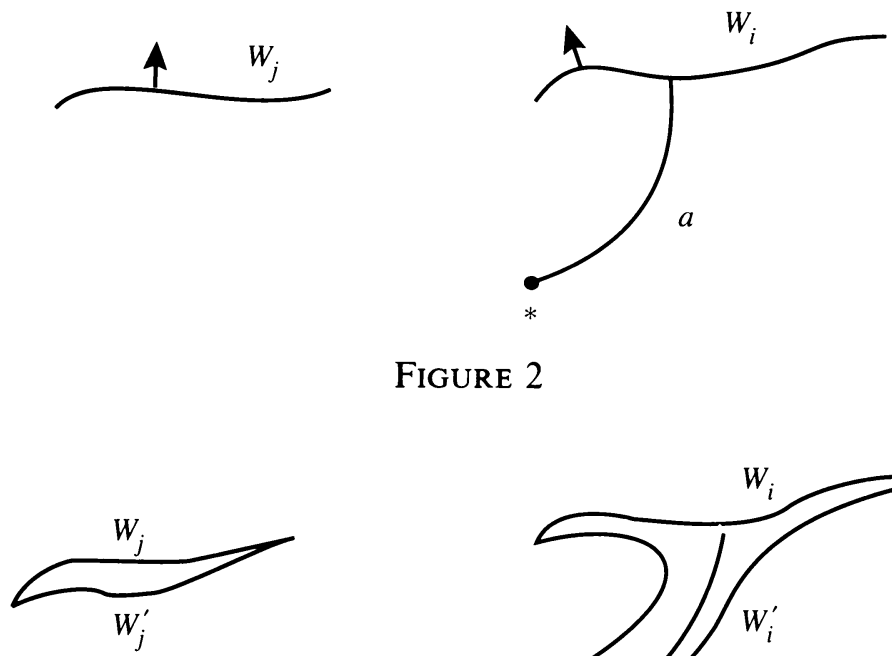

FIGURE 2

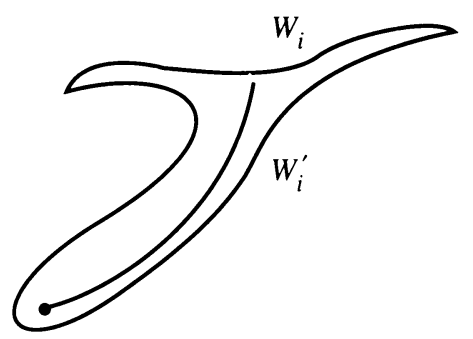

FIGURE 3

Let $W=W_{1} \cup \cdots \cup W_{\mu}$. Join the base point $*$ with $W_{i}$ by a simple arc $a$ approaching $W_{i}$ from the negative side and disjoint from $W_{j}$ for $j \neq i$ (Figure 2). Then construct a new Seifert manifold $W^{\prime}=W_{1}^{\prime} \cup \cdots \cup W_{\mu}^{\prime}$ according to Figure 3.

In order to obtain $W_{j}^{\prime}$ we shift $W_{j}$ slightly in the direction of negative normal, and for $j=i$ we then add a boundary of the thickened arc $a$. It is clear that $W^{\prime}$ is contiguous to $W$ and

$$
f_{W^{\prime}}(x)=t_{i}^{-1} f_{W}(x) t_{i}
$$

for all $x \in \pi_{1}\left(S^{n+2}-\Sigma, *\right)$.

A similar modification with an approaching $W_{i}$ from the positive side and with shifts in the positive normal direction is shown in Figure 4.

In this case we have

$$
f_{W^{\prime}}(x)=t_{i} f_{W}(x) t_{i}^{-1},
$$

where $x \in \pi_{1}\left(S^{n+2}-\Sigma, *\right)$.
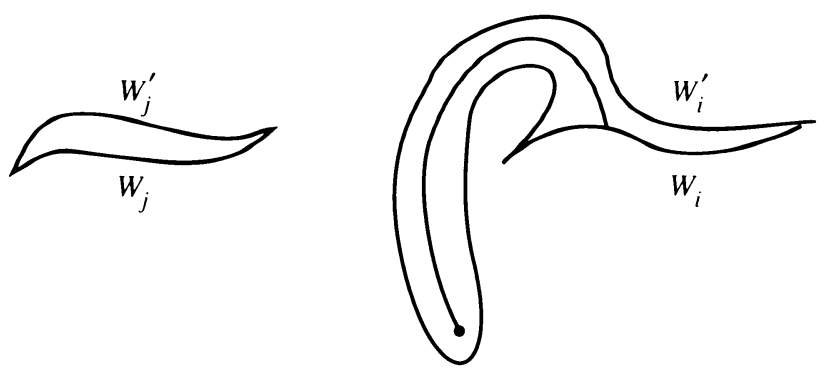

FIGURE 4 
By a number of such modifications we change $W$ (by a chain of contiguities) in such a way that a new Seifert manifold (which we also denote by $W$ ) has the property

$$
f_{V}(x)=f_{W}(x)
$$

for all $x \in \pi_{1}\left(S^{n+2}-\Sigma, *\right)$. Using the arguments of [F3, 1.9-1.12], we may additionally assume that $W_{i}$ coincides with $V_{i}$ in a small tubular neighbourhood of $\Sigma_{i}, i=1, \ldots, \mu$.

Now we can apply the Thom-Pontrjagin construction. Using arguments similar to those in [L3, G, CS], we obtain a submanifold $X \subset S^{n+2} \times[0,1]$ such that

(1) $X$ is compact, $(n+2)$-dimensional, and orientable;

(2) $X$ is a disjoint union of $\mu$ arcwise connected components $X_{1} \cup \cdots \cup X_{\mu}$;

(3) $\partial X_{i}=V_{i} \times 0 \cup W_{i} \times 1 \cup \Sigma_{i} \times[0,1], i=1, \ldots, \mu$;

(4) $V_{i}$ and $W_{i}$ define the same orientation on $X_{i}$.

Using general position arguments we may arrange $X$ to have the following additional property:

(5) Let $h: S^{n+2} \times[0,1] \rightarrow[0,1]$ be the projection; then $\left.h\right|_{X}$ is a nondegenerate Morse function, whose critical values are distinct.

We can also modify $X$ in order to cancel critical points of $\left.h\right|_{X}$ of index 0 and $n+2$. Let $0=c_{0}<c_{1}<\cdots<c_{N}=1$ be a small subdivision of the interval $[0,1]$ such that $\left[c_{\nu}, c_{\nu+1}\right]$ contains at most one critical value of $\left.h\right|_{X}$. Denote

$$
U_{\nu}=h^{-1}\left(c_{\nu}\right) \cap X
$$

$\nu=0,1, \ldots, N$. Then $U_{\nu}$ is a Seifert manifold of $\Sigma$ with $U_{0}=V, U_{N}=$ $W$. If there is no critical point in $\left[c_{i}, c_{i+1}\right]$ then $U_{i+1}$ may be obtained from $U_{i}$ by an orientation-preserving diffeomorphism $f_{i}: S^{n_{+} 2} \rightarrow S^{n+2}$ (i.e., $U_{i+1}=$ $\left.f_{i}\left(U_{i}\right)\right)$. If $\left[c_{i}, c_{i+1}\right]$ contains a critical point then $U_{i+1}$ is obtained from $U_{i}$ by a surgery modification (see Figure 5 ).

It is clear that any such modification can be decomposed into a contiguity and then diffeomorphism. The only possible trouble can occur when the onedimensional handles are glued; it is easy to see that a one-dimensional handle

(1) cannot join two different components of $U_{i}$ and

(2) should approach $U_{i}$ from one side;

otherwise we get a contradiction with conditions (1), (2).

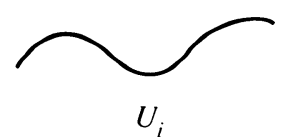

$U_{i}$

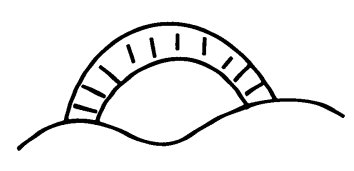

FIGURE 5

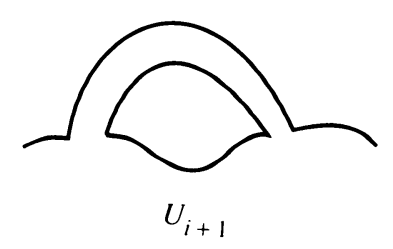

$U_{i+1}$ 
A similar proof in the PL case can be obtained by applying the critical level imbedding technique of [KL].

\section{SEIFERT FORMS AND ISOMETRY STRUCTURES}

2.1. Let $V$ be a Seifert manifold of a boundary link $\Sigma^{n}$ of multiplicity $\mu$. For each $i=1, \ldots, \mu$ fix a base point $x_{i} \in \Sigma_{i}=\partial V_{i}$, where $V=V_{1} \cup \cdots \cup V_{\mu}$. Denote by

$$
\theta_{i i}: V_{i} \wedge V_{i} \rightarrow S^{n+1}
$$

the homotopy Seifert pairing of $V_{i}$ (cf. [F2, p. 186]). For $i \neq j$ let $\theta_{i j}$ be the canonical map

$$
\theta_{i j}: V_{i} \wedge V_{j} \rightarrow S^{n+1}
$$

which is well defined since $V_{i}$ and $V_{j}$ are disjoint subsets of $S^{n+2}$ (cf., for example, [St, Chapter 3]).

We will use the stable homotopy category $\mathrm{Stab}_{0}$, described in [F4, p. 509]. Denote by $\widehat{V}$ the sum (in $\operatorname{Stab}_{0}$ )

$$
\widehat{V}=V_{1} \oplus V_{2} \oplus \cdots \oplus V_{\mu}
$$

The pairings $\theta_{i j}$ define a pairing

$$
\theta: \widehat{V} \otimes \widehat{V} \rightarrow S^{n+1}, \quad \theta=\left(\theta_{i j}\right) .
$$

We also have a collection of $S$-maps

$$
\pi_{i}: \widehat{V} \rightarrow \widehat{V}, \quad i=1, \ldots, \mu,
$$

each $\pi_{i}$ being the composite $\widehat{V} \rightarrow V_{i} \rightarrow \widehat{V}$ of the projection and the inclusion.

The collection $\left(\widehat{V}, \theta, \pi_{1}, \ldots, \pi_{\mu}\right)$ is the Seifert form of $V$.

2.2. Lemma. Any Seifert form $\left(\widehat{V}, \theta, \pi_{1}, \ldots, \pi_{\mu}\right)$ has the following properties:

(1) $\theta+(-1)^{n+1} \theta^{\prime}: \widehat{V} \otimes \widehat{V} \rightarrow S^{n+1}$ is a Spanier-Whitehead duality;

(2) $\theta \circ\left(\pi_{i} \otimes \pi_{j}\right)=(-1)^{n} \theta^{\prime} \circ\left(\pi_{i} \otimes \pi_{j}\right)$ for $i \neq j$;

(3) $\pi_{i} \circ \pi_{j}=\delta_{i j} \pi_{j}, \pi_{1}+\pi_{2}+\cdots+\pi_{\mu}=1_{\widehat{V}}$.

Here $\theta^{\prime}$ denotes $\theta \circ T$, where $T: \widehat{V} \otimes \widehat{V} \rightarrow \widehat{V} \otimes \widehat{V}$ is the interchanging map, and $\delta_{i j}$ is Kronecker's delta.

Proof. Statements (2) and (3) are evident. Statement (1) may be deduced from [F1, Theorem 1.4] in the following way. Consider a small $(n+1)$-dimensional disk $D^{n+1}$ imbedded in $S^{n+2}-V$. Connect it by a smooth $(n+1)$-dimensional band $B_{i}$ with $V_{i}, i=1, \ldots, \mu$. We assume that $B_{i}=D_{i}^{n} \times I, B_{i} \cap V_{i}=$ $B_{i} \cap \partial V_{i}=D_{i}^{n} \times\{0\}, B_{i} \cap D^{n+1}=B_{i} \cap \partial D^{n+1}=D_{i}^{n} \times\{1\}$, and $B_{i} \cap V_{j}=\varnothing=$ $B_{i} \cap B_{j}$ for $i \neq j$. The union $W=V_{1} \cup \cdots \cup V_{\mu} \cup B_{1} \cup \cdots \cup B_{\mu} \cup D^{n+1}$ is a Seifert manifold of a spherical knot in $S^{n+2}$ and it is clear that there is a homotopy equivalence $\varphi: W \rightarrow \widehat{V}$ such that $\theta \circ(\varphi \otimes \varphi)$ is the homotopy Seifert pairing of the knot. Statement (1) now follows from [F2, Theorem 1.4].

2.3. An abstract Seifert form of dimension $n$ and multiplicity $\mu$ is a collection $\left(X, \theta, \pi_{1}, \ldots, \pi_{\mu}\right)$, where $X$ is a stable (or virtual) complex (i.e., an object 
of $\left.\mathrm{Stab}_{0}\right), \theta: X \otimes X \rightarrow S^{n+1}$ is an $S$-map with $\theta+(-1)^{n+1} \theta^{\prime}$ a duality, and $\pi_{1}, \pi_{2}, \ldots, \pi_{\mu}$ are $S$-maps $X \rightarrow X$ satisfying

$$
\pi_{i} \circ \pi_{j}=\delta_{i j} \pi_{j}, \quad \pi_{1}+\cdots+\pi_{\mu}=1_{X},
$$

and

$$
\theta \circ\left(\pi_{i} \otimes \pi_{j}\right)=(-1)^{n} \theta^{\prime} \circ\left(\pi_{i} \otimes \pi_{j}\right)
$$

for $i \neq j$. Two abstract Seifert forms are isomorphic if there exists a stable homotopy equivalence between the corresponding spaces, commuting with the $\pi_{i}$ 's and transforming one pairing $\theta$ into the other.

2.4. Theorem. Suppose $r$ and $n$ are integers with $3 r \geq n+1 \geq 6$. Then:

(1) Any abstract Seifert form $\left(X, \theta, \pi_{1}, \ldots, \pi_{\mu}\right)$ of dimension $n$ with length $(X) \leq n-2 r$ (cf. [F4, p. 510]) is isomorphic to the Seifert form of an $r$-simple Seifert manifold $V$ of a boundary $\mu$-component link $\Sigma^{n}$.

(2) Assume that $\Sigma^{n}$ and $\Sigma^{\prime \prime}$ are two r-simple $\mu$-component links and, $V$ and $V^{\prime}$ are the corresponding $r$-simple Seifert manifolds. If the Seifert forms of $V$ and $V^{\prime}$ are isomorphic then there exists an isotopy of $S^{n+2}$ taking each $V_{i}$ onto $V_{i}^{\prime}, i=1, \ldots, \mu$, and preserving the orientations.

The proof is given in $\S 2.9$ below.

2.5. Another stable homotopy object (which is in fact equivalent to the abstract Seifert form but will be more convenient later) is the stable isometry structure. A stable isometry structure of dimension $n$ and multiplicity $\mu$ is a collection

$$
\left(X, u, z, \pi_{1}, \ldots, \pi_{\mu}\right),
$$

where $X$ is a virtual complex and $u: X \otimes X \rightarrow S^{n+1}, z: X \rightarrow X$, and $\pi_{i}: X \rightarrow X, i=1, \ldots, \mu$ are $S$-maps, satisfying:

(1) $u$ is a Spanier-Whitehead duality;

(2) $u^{\prime}=(-1)^{n+1} u$;

(3) $u \circ\left(z \otimes 1_{X}\right)=u \circ\left(1_{X} \otimes \bar{z}\right)$, where $\bar{z}=1_{X}-z$;

(4) $u \circ\left(\pi_{i} \otimes \pi_{j}\right)=0$ for $i \neq j$;

(5) $\pi_{i} \circ \pi_{j}=\delta_{i j} \pi$;

(6) $\pi_{1}+\cdots+\pi_{\mu}=1_{X}$.

The notion of isomorphism of stable isometry structures can be introduced in an obvious way.

Any stable isometry structure $\left(X, u, z, \pi_{1}, \ldots, \pi_{\mu}\right)$ defines an abstract Seifert form $\left(X, \theta, \pi_{1}, \ldots, \pi_{\mu}\right)$, where $\theta=u \circ(1 \otimes z)$. Conversely, given an abstract Seifert form $\left(X, \theta, \pi_{1}, \ldots, \pi_{\mu}\right)$ one can define $u: X \otimes X \rightarrow S^{n+1}$ by $u=\theta+(-1)^{n+1} \theta^{\prime}$; this is a Spanier-Whitehead duality and so there exists a unique $S$-map $z: X \rightarrow X$ such that $\theta=u \circ(1 \otimes z)$. It is easy to see that the collection $\left(X, u, z, \pi_{1}, \ldots, \pi_{\mu}\right)$ is a stable isometry structure.

The following statement is just another version of Theorem 2.4.

2.6. Theorem. Suppose $r$ and $n$ are integers with $3 r \geq n+1 \geq 6$. There is a one-to-one correspondence between the set of isotopy types of embeddings into $S^{n+2}$ of $r$-simple $\mu$-component Seifert manifolds and the set of isomorphism classes of stable isometry structures $\left(X, u, z, \pi_{1}, \ldots, \pi_{\mu}\right)$ of dimension $n$ with length $(X) \leq n-2 r$. 
2.7. A configuration (of dimension $n$ and multiplicity $\mu$ ) is a collection $\left(X_{i} ; v_{i j}\right), i, j=1, \ldots, \mu$, where $X_{i}$ is a virtual complex (i.e., an object of $\left.\mathrm{Stab}_{0}\right), v_{i j}: X_{i} \otimes X_{j} \rightarrow S^{n+1}$, defined for $i \neq j, i, j \in\{1, \ldots, \mu\}$, such that

$$
v_{i j}=(-1)^{n} v_{j i}^{\prime},
$$

where $v_{j i}^{\prime}$ denotes $v_{j i} \circ T, T: X_{i} \otimes X_{j} \rightarrow X_{j} \otimes X_{i}$ being the "interchanging" map. Two configurations $\left(X_{i}, v_{i j}\right)$ and $\left(Y_{i}, w_{i j}\right)$ of the same dimension and multiplicity are isomorphic if there exists a family of $S$-equivalences $f_{i}: X_{i} \rightarrow$ $Y_{i}, i=1, \ldots, \mu$, with the property

$$
w_{i j} \circ\left(f_{i} \otimes f_{j}\right)=v_{i j}
$$

for all $i, j=1, \ldots, \mu, i \neq j$.

Let $\left(X_{i}, v_{i j}\right)$ be a configuration of dimension $n$ and multiplicity $\mu$. Its geometric realization is a sequence of compact $(n+2)$-dimensional smooth submanifolds $N_{1}, \ldots, N_{\mu}$ of $S^{n+2}$ supplied with a family of $S$-equivalences

$$
f_{i}: X_{i} \rightarrow N_{i}, \quad i=1, \ldots, \mu,
$$

such that

$$
v_{i j}=w_{i j} \circ\left(f_{i} \otimes f_{j}\right)
$$

for all $i \neq j, i, j=1, \ldots, \mu$, where $w_{i j}: N_{i} \otimes N_{j} \rightarrow S^{n+1}$ is the canonical pairing (arising from the embedding of $N_{i}$ and $N_{j}$ as disjoint subsets of $S^{n+2}$ ). Two geometric realizations $\left(N_{i}, f_{i}\right)$ and $\left(N_{i}^{\prime}, f_{i}^{\prime}\right)$ will be called homeomorphic if there exists a diffeomorphism $h: S^{n+2} \rightarrow S^{n+2}$ (which is isotopic to the identity) such that

$$
h\left(N_{i}\right)=N_{i}^{\prime}, \quad i=1, \ldots, \mu,
$$

and the diagram

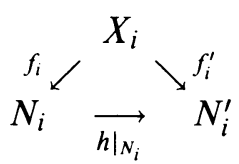

commutes for all $i=1, \ldots, \mu$ in stable homotopy.

2.8. Proposition. Let $r$ and $n$ be integers with $3 r \geq n+1 \geq 6$. Any configuration $\left(X_{i}, v_{i j}\right)$ of dimension $n$, with each $X_{i}$ being r-connected and $(n-r)$ dimensional, admits a geometric realization which is unique up to homeomorphism.

Proof. For $\mu=1$ this statement follows immediately from Wall's theory [W]. For $\mu>1$ one may use the following arguments. Suppose we have a configuration $\left(X_{i}, v_{i j}\right)$ and we have constructed $N_{1}, \ldots, N_{k-1}$ and $f_{i}: X_{i} \rightarrow N_{i}$, $i=1, \ldots, k-1$, with the desired properties. Pick an $(n+2)$-dimensional ball $B \subset S^{n+2}, B \cap N_{i}=\varnothing, i=1, \ldots, k-1$, and join each $N_{i}$ with $B$ by nonintersecting 1-handles. Let $N$ denote the union of $N_{1}, \ldots, N_{k-1}$ with these 1 -handles and with $B$. If $Y$ denotes $S^{n+2}-$ int $N$, then according to the Spanier-Whitehead duality theory, stable homotopy classes $X_{k} \rightarrow Y$ correspond bijectively to systems of pairings

$$
\left\{v_{k i}: X_{k} \otimes X_{i} \rightarrow S^{k+1} ; i=1, \ldots, k-1\right\} .
$$

Thus if we are given $\left\{v_{k i}\right\}$ for $i<k$ we can find a unique stable homotopy class $f: X_{k} \rightarrow Y$ corresponding to it. Using our dimension and connectivity 
assumptions we may realize $f$ by a map (which is unique due to the suspension theorem). Now at the last stage we can apply the thickening theory of Wall [W], similarly to the case of knots [F2, F4].

Uniqueness of realization follows similarly.

2.9. Proof of Theorem 2.4(1). If we are given an abstract Seifert form $\left(X, \theta, \pi_{1}, \ldots, \pi_{\mu}\right)$ then we can apply the Splitting of Homotopy Idempotents Theorem of [Fr] to conclude that $X$ can be identified with the direct sum $X_{1} \oplus X_{2} \oplus \cdots \oplus X_{\mu}$ in such a way that $\pi_{i}=q_{i} \circ p_{i}$, where $p_{i}: X \rightarrow X_{i}$ and $q_{i}: X_{i} \rightarrow X$ are the projection and inclusion maps, respectively.

Consider the configuration $\left\{X_{i}, v_{i j}\right\}$, where

$$
v_{i j}=\theta\left(q_{i} \otimes q_{j}\right), \quad i, j \in\{1, \ldots, \mu\}, i \neq j .
$$

By Proposition 2.8 there exists a geometric realization $N_{1}, \ldots, N_{\mu}$ of $\left\{X_{i}, v_{i j}\right\}$. For each $i=1, \ldots, \mu$ we have an $(n+2)$-dimensional submanifold $N_{i} \subset S^{n+2}$ and a stable homotopy equivalence $f_{i}: N_{i} \rightarrow X_{i}$. Our initial data also give us a pairing

$$
\theta_{i}=\theta \circ\left(q_{i} \otimes q_{i}\right): X_{i} \otimes X_{i} \rightarrow S^{n+1} .
$$

Using the arguments of the proof of Theorem 1.3 of [F2] (cf. 1.10 of [F2]), one gets an $r$-connected $(n+1)$-dimensional manifold $V_{i} \subset \partial N_{i}$ with $\partial V_{i}$ a homotopy sphere and with the property that the inclusion $b_{i}: V_{i} \rightarrow N_{i}$ is an equivalence and $\theta_{i} \circ\left(f_{i} \circ b_{i} \otimes f_{i} \circ b_{i}\right)$ is the homotopy Seifert pairing of $V_{i}$. It is now clear that the union

$$
V=V_{1} \cup \cdots \cup V_{\mu}
$$

is the desired Seifert manifold of a boundary link $\Sigma=\partial V$.

Statement (2) can be proved similarly (using the arguments of the proof of Theorem 1.2 in $[\mathrm{F} 2])$.

\section{Almost closed Seifert surfaces}

The aim of this and the next sections is to describe in terms of the stable homotopy theory the precise relation between stable homotopy structures of different Seifert manifolds of a link. On the level of homology this has been done by J. Levine [L3] in terms of $S$-equivalence of Seifert matrices. A more invariant approach was suggested in [F2, F3] under the name of $R$-equivalence. The present exposition will be a generalization of $[F 2, F 3, F 4]$ to the case of links.

Almost closed Seifert manifolds, which will be studied in the present section, are used in the next section to measure the difference between different Seifert manifolds of a link.

3.1. Proposition. There is a canonical construction which assigns to any configuration $\left(X_{i}, v_{i j}\right)$ a stable isometry structure $S\left(X_{i}, v_{i j}\right)$ (of the same dimension and multiplicity) with the following properties:

(1) Let $\left(N_{i}, f_{i}\right)$ be a geometric realization of $\left(X_{i}, v_{i j}\right)$, let $V_{i}$ be obtained from $\partial N_{i}$ by removing a small $(n+1)$-dimensional disk in $\partial N_{i}$, and orient $V_{i}$ by directing the positive normal outside $N_{i}$. Then $V=V_{1} \cup \cdots \cup V_{\mu}$ is a Seifert manifold (of the trivial link) and its stable isometry structure is isomorphic to $S\left(X_{i}, v_{i j}\right)$. 
(2) Stable isometry structures $S\left(X_{i}, v_{i j}\right)$ and $S\left(Y_{i}, w_{i j}\right)$ are isomorphic if and only if the configurations $\left(X_{i}, v_{i j}\right)$ and $\left(Y_{i}, w_{i j}\right)$ are isomorphic.

(3) $A$ stable isometry structure $\left(X, u, z, \pi_{1}, \ldots, \pi_{\mu}\right)$ can be obtained as $S\left(X_{i}, v_{i j}\right)$ for some configuration $\left(X_{i}, v_{i j}\right)$ if and only if $\bar{z} \pi_{k} z=0$ for all $k=1, \ldots, \mu$.

Any Seifert manifold $V=V_{1} \cup \cdots \cup V_{\mu}$ which can be obtained by the construction described in Proposition 3.1(1) will be called almost closed.

Proof. The construction of $S\left(X_{i}, v_{i j}\right)$ proceeds as follows. For each $i=$ $1, \ldots, \mu$ let $u_{i}: X_{i} \otimes Z_{i} \rightarrow S^{n+1}$ be a Spanier-Whitehead duality. For $i \neq j$ there is a unique $S$-map $\varphi_{j i}: X_{j} \rightarrow Z_{i}$ with the property that

$$
u_{i} \circ\left(1_{X_{i}} \otimes \varphi_{j i}\right)=v_{i j} .
$$

Denote by $X$ the direct sum

$$
X=X_{1} \oplus \cdots \oplus X_{\mu} \oplus Z_{1} \oplus \cdots \oplus Z_{\mu}
$$

and define $u: X \otimes X \rightarrow S^{n+1}$ by

$$
\begin{gathered}
\left.u\right|_{X_{i} \otimes X_{j}}=0,\left.\quad u\right|_{X_{i} \otimes Z_{j}}=\delta_{i j} u_{i}, \\
\left.u\right|_{Z_{i} \otimes X_{j}}=(-1)^{n+1} \delta_{i j} u_{i}^{\prime},\left.\quad u\right|_{Z_{i} \otimes Z_{j}}=0,
\end{gathered}
$$

where $\delta_{i j}$ is the Kronecker symbol. The next object $z: X \rightarrow X$ will be defined by

$$
\left.z\right|_{X_{i}}=0,\left.\quad z\right|_{Z_{i}}=\mathrm{id}_{Z_{i}}: Z_{i} \rightarrow Z_{i},
$$

where $\operatorname{id}_{Z_{i}}$ is the identity map, $i=1, \ldots, \mu$. We define the $S$-map $\pi_{k}: X \rightarrow$ $X$ by the formulae

$$
\begin{gathered}
\pi_{\left.k\right|_{x_{i}}}= \begin{cases}\operatorname{id}_{X_{k}}+\sum_{j \neq k} \varphi_{k j} & \text { for } i=k, \\
-\varphi_{i k} & \text { for } i \neq k,\end{cases} \\
\pi_{k} \mid Z_{i}= \begin{cases}0 & \text { for } i \neq k, \\
\operatorname{id}_{Z_{k}} & \text { for } i=k .\end{cases}
\end{gathered}
$$

A trivial check (which is omitted) shows that the collection

$$
\left(X, u, z, \pi_{1}, \ldots, \pi_{\mu}\right)
$$

is a stable isometry structure, and satisfies $\bar{z} \pi_{k} z=0$ for all $k=1, \ldots, \mu$.

To prove the last statement of the proposition, assume that the collection $\left(X, u, z, \pi_{1}, \ldots, \pi_{\mu}\right)$ is an arbitrary stable isometry structure with $\bar{z} \pi_{k} z=0$ for all $k=1, \ldots, \mu$. Consider $S$-maps

$$
P_{i}: X \rightarrow X, \quad i=1, \ldots, 2 \mu,
$$

defined by

It is clear that

$$
P_{i}= \begin{cases}\bar{z} \pi_{i} & \text { for } i=1, \ldots, \mu \\ \pi_{i-\mu} z & \text { for } i=\mu+1, \ldots, 2 \mu .\end{cases}
$$

$$
P_{i} P_{j}=\delta_{i j} P_{j}, \quad \sum_{i=1}^{2 \mu} P_{i}=1_{X} .
$$


By the Freyd theorem [Fr] there is a direct sum decomposition

$$
X \underset{q_{i}}{\stackrel{p_{i}}{\rightleftarrows}} X_{i}
$$

with

$$
q_{i} \circ p_{i}=P_{i}, \quad p_{i} \circ q_{i}=\mathrm{id}_{X_{i}}, \quad i=1,2, \ldots, 2 \mu .
$$

Define $v_{i j}: X_{i} \otimes X_{j} \rightarrow S^{n+1}$ by

$$
v_{i j}=u \circ\left(\pi_{i} q_{i} \otimes z \pi_{j} q_{j}\right)
$$

for $i \neq j, i, j=1, \ldots, \mu$.

We thus obtain a configuration $\left(X_{i}, v_{i j}\right)$ and we want to prove that the original stable isometry structure $\left(X, u, z, \pi_{1}, \ldots, \pi_{\mu}\right)$ is isomorphic to $S\left(X_{i}, v_{i j}\right)$. The identity

$$
\begin{aligned}
u\left(q_{i} \otimes q_{j}\right) & =u\left(q_{i} \otimes q_{j} \circ p_{j} \circ q_{j}\right)=u\left(q_{i} \otimes P_{j} \circ q_{j}\right) \\
& =u\left(P_{\bar{j}} \circ q_{i} \otimes q_{j}\right)=\delta_{i \bar{j}} u\left(q_{i} \otimes q_{j}\right)
\end{aligned}
$$

where

$$
\bar{j}= \begin{cases}j+\mu & \text { for } j \leq \mu, \\ j-\mu & \text { for } j>\mu,\end{cases}
$$

shows that $u\left(q_{i} \otimes q_{j}\right)=0$ unless $|i-j|=\mu$. It follows that

$$
u_{i}=u \circ\left(q_{i} \otimes q_{i+\mu}\right): X_{i} \otimes X_{i+\mu} \rightarrow S^{n+1}, \quad i=1, \ldots, \mu,
$$

is a duality map, and thus we can use it in the construction of $S\left(X_{i}, v_{i j}\right)$ instead of $u_{i}: X_{i} \otimes Z_{i} \rightarrow S^{n+1}$. It is easy to show that the maps

$$
\varphi_{j i}: X_{j} \rightarrow X_{i+\mu}, \quad i \neq j, i, j=1, \ldots, \mu,
$$

which appear in the construction of $S\left(X_{i}, v_{i j}\right)$, are given by the formula

$$
\varphi_{j i}=P_{i+\mu} \circ \pi_{j} \circ q_{j},
$$

and the map $Q: X_{1} \oplus \cdots \oplus X_{2 \mu} \rightarrow X, Q=\sum_{i=1}^{2 \mu} q_{i}$, gives an isomorphism between $S\left(X_{i}, v_{i j}\right)$ and $\left(X, u, z, \pi_{1}, \ldots, \pi_{\mu}\right)$.

This completes the proof of statement (3).

Statement (2) follows from the arguments used in the proof of (3): we have shown that all objects in $\left(X, u, z, \pi_{1}, \ldots, \pi_{\mu}\right)$ could be uniquely determined in terms of $X_{i}, v_{i j}$ and vice versa.

Now we have to prove statement (1).

Let $N_{1}, \ldots, N_{\mu} \subset S^{n+2}$ be a set of compact smooth $(n+2)$-dimensional pairwise disjoint submanifolds and let

$$
v_{i j}: N_{i} \otimes N_{j} \rightarrow S^{n+1}, \quad i \neq j,
$$

be the corresponding pairing, $i, j=1, \ldots, \mu$. We construct $V_{i}$ by removing from $\partial N_{i}$ a small $(n+1)$-dimensional disk and we have to show that the stable isometry structure

$$
\left(\widehat{V}, u, z, \pi_{1}, \ldots, \pi_{\mu}\right)
$$

corresponding to $V=V_{1} \cup V_{2} \cup \cdots \cup V_{\mu}$ is isomorphic to $S\left(N_{i}, v_{i j}\right)$.

Let $B^{n+2}$ be a small $(n+2)$-dimensional ball in $S^{n+2}$ disjoint from $N_{i}, i=$ $1, \ldots, \mu$. Join each $N_{i}$ to $B$ by a 1 -handle $H_{i}$; in other words, $H_{i}=D^{n+1} \times$ 
$[0,1], H_{i} \cap N_{i}=H_{i} \cap \partial N_{i}=D^{n+1} \times 0, H_{i} \cap B=H_{i} \cup \partial B=D^{n+1} \times 1, H_{i} \cap H_{j}=\varnothing$ for $i \neq j$. Denote by $N$ the union $N_{1} \cup \cdots \cup N_{\mu} \cup H_{1} \cup \cdots \cup H_{\mu} \cup B$. Denote by $Y$ the complement $S^{n+2}-\operatorname{int} N$. The space $\widehat{V}$ can be naturally identified with the result of removing from $\partial N$ a small $(n+1)$-dimensional disk.

By Proposition 2.1 of [F2] there exists a direct sum decomposition

$$
Y \underset{\alpha}{\stackrel{j}{\leftrightarrows}} \widehat{V} \underset{\beta}{\stackrel{i}{\leftrightarrows}} N
$$

(where $i: \widehat{V} \rightarrow N$ and $j: \widehat{V} \rightarrow Y$ are the inclusions) with the property

$$
z=\alpha \circ j, \quad \bar{z}=\beta \circ i
$$

(cf. also Corollary 2.2 in [F2] and Lemma 2.9 in [F4]). Identifying $N$ with the wedge of $N_{1} \vee \cdots \vee N_{\mu}$, we have another direct sum decomposition

$$
N \underset{q_{k}}{\stackrel{p_{k}}{\rightleftarrows}} N_{k}, \quad k=1, \ldots, \mu .
$$

It is clear from the geometry that

$$
i \circ \pi_{k}=q_{k} \circ p_{k} \circ i, \quad k=1, \ldots, \mu,
$$

and thus

$$
\beta \circ q_{k} \circ p_{k} \circ i=\beta i \pi_{k}=\bar{z} \pi_{k} .
$$

This shows that the projector $P_{k}: \widehat{V} \rightarrow \widehat{V}$ corresponding to the summand

$$
\widehat{V} \underset{\beta q_{k}}{\stackrel{p_{k} i}{\rightleftarrows}} N_{k}
$$

is given by the formula $P_{k}=\bar{z} \pi_{k}$ which agrees with the construction of $S\left(N_{i}, v_{i j}\right)$. We also have

$$
\bar{z} \pi_{k} z=P_{k} z=\beta q_{k} p_{k} i \alpha j=0
$$

since $i \alpha=0$. To complete the proof of statement (1) we only have to show that

$$
v_{k l}=u \circ\left(\pi_{k} \beta q_{k} \otimes z \pi_{l} \beta q_{l}\right)
$$

for $k \neq l, \quad k, l=1, \ldots, \mu$. According to the definition $u \circ(1 \otimes z)=\theta$, where $\theta: \widehat{V} \otimes \widehat{V} \rightarrow S^{n+1}$ is the Seifert form (cf. §2.5) and

$$
\theta \circ\left(\pi_{k} \otimes \pi_{l}\right)=v_{k l} \circ\left(p_{k} \circ i \otimes p_{l} \circ i\right)
$$

(obvious). Thus

$$
\begin{aligned}
u \circ\left(\pi_{k} \beta q_{k} \otimes z \pi_{l} \beta q_{l}\right) & =\theta \circ\left(\pi_{k} \otimes \pi_{l}\right) \circ\left(\beta q_{k} \otimes \beta q_{l}\right) \\
& =v_{k l} \circ\left(p_{k} i \otimes p_{l} i\right) \circ\left(\beta q_{k} \otimes \beta q_{l}\right) \\
& =v_{k l} \circ\left(p_{k} i \beta q_{k} \otimes p_{l} i \beta q_{l}\right)=v_{k l}
\end{aligned}
$$

since

$$
p_{k} i \beta q_{k}=\operatorname{id}_{N_{k}}, \quad p_{l} i \beta q_{l}=\operatorname{id}_{N_{l}} .
$$

This completes the proof. 
3.2. Corollary. (1) If $\left(\widehat{V}, u, z, \pi_{1}, \ldots, \pi_{\mu}\right)$ is the stable isometry structure of an almost closed Seifert manifold, then $\bar{z} \pi_{k} z=0$ for all $k=1,2, \ldots, \mu$.

(2) Let $V$ be an $r$-simple Siefert manifold of an $n$-dimensional link, where $3 r \geq n+1 \geq 6$. If $\bar{z} \pi_{k} z=0$ for all $k \in\{1, \ldots, \mu\}$, then $V$ is almost closed. Proof. (1) follows from statements (1) and (3) of Proposition 3.1. (2) follows from Propositions 2.8 and 3.1 and Theorem 2.6.

\section{THE CLASSIFICATION OF $\mathscr{F}$-LINKS}

In this section we will describe the relation between stable isometry structures of contiguous Seifert manifolds. Under stability assumptions it will be a complete description. Thus, combining this result with Theorem 1.6 we obtain a stable-homotopy classification of stable $\mathscr{F}$-links. In the case of $(q-1)$-simple $(2 q-1)$-dimensional links it gives an algebraic classification.

4.1. Let $\mathfrak{q}_{1}=\left(X_{1}, u_{1}, z_{1}, \pi_{1}, \ldots, \pi_{\mu}\right)$ and $\mathfrak{q}_{2}=\left(X_{2}, u_{2}, z_{2}, \tilde{\pi}_{1}, \ldots, \tilde{\pi}_{\mu}\right)$ be two stable isometry structures of dimension $n$. We will say that $q_{1}$ is contiguous to $\mathfrak{q}_{2}$ if there exist $S$-maps

$$
\varphi: X_{1} \rightarrow X_{2}, \quad \psi: X_{2} \rightarrow X_{1}
$$

with

(a) $u_{2} \circ\left(1_{X_{2}} \otimes \varphi\right)=u_{1} \circ\left(\psi \otimes 1_{X_{1}}\right)$;

(b) $\varphi \circ \pi_{i} \circ z_{1}=z_{2} \circ \tilde{\pi}_{i} \circ \varphi$;

(c) $\psi \circ \tilde{\pi}_{i} \circ \bar{z}_{2}=\bar{z}_{1} \circ \pi_{i} \circ \psi$;

(d) $\varphi \circ \pi_{i} \circ \psi=z_{2} \circ \tilde{\pi}_{i} \circ \bar{z}_{2}$

(e) $\psi \circ \tilde{\pi}_{i} \circ \varphi=\bar{z}_{1} \circ \pi_{i} \circ z_{1}$, for all $i=1,2, \ldots, \mu$.

This definition generalizes the corresponding notion for knots (cf. [F2, F4]).

Each stable isometry structure is contiguous to itself (one may take $\varphi=$ $z, \psi=\bar{z})$.

If $\mathfrak{q}=\left(X, u, z, \pi_{1}, \ldots, \pi_{\mu}\right)$ is an isometry structure, we may define $-\mathfrak{q}$ as $\left(X,-u, \bar{z}, \pi_{1}, \ldots, \pi_{\mu}\right)$. If $\mathfrak{q}_{1}$ is contiguous to $\mathfrak{q}_{2}$, then $-\mathfrak{q}_{2}$ is contiguous to $-q_{1}$.

The following theorem is the main result of this section.

4.2. Theorem. (1) Let $V$ and $W$ be two Seifert manifolds of a boundary link $\Sigma^{n}$ in $S^{n+2}$ with $V$ contiguous to $W$. Then the stable isometry structure $\mathfrak{q}_{V}=\left(\widehat{V}, u_{V}, z_{V}, \pi_{1}^{V}, \ldots, \pi_{\mu}^{V}\right)$, corresponding to $V$, is contiguous to the stable isometry structure $\mathfrak{q}_{W}=\left(\widehat{W}, u_{W}, z_{W}, \pi_{1}^{W}, \ldots, \pi_{\mu}^{W}\right)$ corresponding to $W$.

(2) Let $r, n$ be integers with $3 r \geq n+1 \geq 6$. Let $V$ and $W$ be $r$-simple Seifert manifolds of boundary links $\Sigma_{V}=\partial V$ and $\Sigma_{W}=\partial W$, and let $\mathfrak{q}_{V}$ and $\mathfrak{q}_{W}$ be the corresponding stable isometry structures. If $\mathfrak{q}_{V}$ is contiguous to $\mathfrak{q}_{W}$, then there exists an isotopy of $S^{n+2}$ taking $W$ onto another Siefert manifold $W^{\prime}$ such that $V$ is contiguous to $W^{\prime}$; in particular, the links $\Sigma_{V}$ and $\Sigma_{W}$ are equivalent.

Proof. (1) Assume that $V$ is contiguous to $W$. Denote by $N_{i}$ the closure of the arcwise connected component of $S^{n+2}-\left(V_{i} \cup W_{i}\right)$ with the positive normal to $W_{i}$ pointing inside $N_{i}$. We have compact connected disjoint $(n+2)$-dimensional submanifolds $N_{1}, \ldots, N_{\mu}$ in $S^{n+2}$ and we can form an almost closed Seifert 
manifold $U=U_{1} \cup \cdots \cup U_{\mu}$, where $U_{i}$ is obtained from $\partial N_{i}$ by removing a small $(n+1)$-dimensional disk (cf. the previous section). Orient $U$ according to $V$.

Let $\mathfrak{q}_{U}=\left(\widehat{U}, u_{U}, z_{U}, \pi_{1}^{U}, \ldots, \pi_{\mu}^{U}\right)$ be the stable isometry structure of $U$. It is clear that there is natural identification of $\widehat{U}$ with $\widehat{V} \oplus \widehat{W}$, and the Seifert form $\theta_{U}$ of $U$ has the matrix presentation

$$
\left[\begin{array}{cc}
\theta_{V} & \alpha \\
(-1)^{n} \alpha^{\prime} & (-1)^{n} \theta_{W}^{\prime}
\end{array}\right]
$$

where $\alpha: \widehat{V} \otimes \widehat{W} \rightarrow S^{n+1}$ is the canonical pairing and the prime denotes transposition (cf. [F2, F4]). Therefore, we have

$$
\begin{gathered}
u_{U}=\theta_{U}+(-1)^{n+1} \theta_{U}^{\prime}=\left[\begin{array}{cc}
u_{V} & 0 \\
0 & -u_{W}
\end{array}\right], \\
z_{U}=\left[\begin{array}{cc}
z_{V} & \varphi \\
\psi & \bar{z}_{W}
\end{array}\right], \quad \pi_{i}^{U}=\left[\begin{array}{cc}
\pi_{i}^{V} & 0 \\
0 & \pi_{i}^{W}
\end{array}\right],
\end{gathered}
$$

where $\varphi: \widehat{V} \rightarrow \widehat{W}$ and $\psi: \widehat{W} \rightarrow \widehat{V}$ are $S$-maps with

$$
\alpha=u_{V} \circ\left(1_{V} \otimes \psi\right)=u_{W} \circ\left(\varphi \otimes 1_{W}\right) .
$$

From Corollary 2.6 we know that

$$
\bar{z}_{U} \pi_{i}^{U} z_{U}=0, \quad i=1, \ldots, \mu .
$$

Thus we have

$$
\left[\begin{array}{cc}
\bar{z}_{V} & -\varphi \\
\psi & z_{W}
\end{array}\right] \circ\left[\begin{array}{cc}
\pi_{i}^{V} & 0 \\
0 & \pi_{i}^{W}
\end{array}\right] \circ\left[\begin{array}{cc}
z_{V} & \varphi \\
\psi & \bar{z}_{W}
\end{array}\right]=0
$$

and this matrix equation gives the following four equalities:

$$
\begin{aligned}
& \varphi \pi_{i}^{V} z_{V}=z_{W} \pi_{i}^{W} \varphi, \quad \psi \pi_{i}^{W} \bar{z}_{W}=\bar{z}_{V} \pi_{i}^{V} \varphi, \\
& \psi \pi_{i}^{2} \varphi=\bar{z}_{V} \pi_{i}^{V} z_{V}, \quad \varphi \pi_{i}^{V} \psi=z_{W} \pi_{i}^{W} \bar{z}_{W},
\end{aligned}
$$

which proves (1).

Let us now prove the second statement of the theorem. Assume that $\mathfrak{q}_{V}$ is contiguous to $\mathfrak{q}_{W}$ and let $\varphi: \widehat{V} \rightarrow \widehat{W}, \psi: \widehat{W} \rightarrow \widehat{V}$ be the maps satisfying conditions $(\mathrm{a})-(\mathrm{e})$ of 4.1. Define a new stable isometry structure $\mathfrak{q}_{U}=$ $\left(\widehat{U}, u_{U}, z_{U}, \pi_{1}^{U}, \ldots, \pi_{\mu}^{U}\right)$, where

$$
\widehat{U}=\widehat{V} \oplus \widehat{W}, \quad u_{U}=\left[\begin{array}{cc}
u_{V} & 0 \\
0 & -u_{W}
\end{array}\right], \quad z_{U}=\left[\begin{array}{cc}
z_{V} & \varphi \\
\psi & \bar{z}_{W}
\end{array}\right],
$$

and

$$
\pi_{i}^{U}=\left[\begin{array}{cc}
\pi_{i}^{V} & 0 \\
0 & \pi_{i}^{W}
\end{array}\right]
$$

Conditions (a)-(e) of 4.1 are equivalent to the statement that the collection $q_{U}$ is a stable isometry structure with the property $\bar{z}_{U} \pi_{i}^{U} z_{U}=0$ for all $i=$ $1, \ldots, \mu$.

Let us define $S$-maps $P_{i}: \widehat{U} \rightarrow \widehat{U}, i=1, \ldots, 2 \mu$, by

$$
\begin{aligned}
& P_{i}: \widehat{U} \rightarrow V_{i} \rightarrow \widehat{U}, \quad i=1, \ldots, \mu, \\
& P_{i}: \widehat{U} \rightarrow W_{i} \rightarrow \widehat{U}, \quad i=\mu+1, \ldots, 2 \mu,
\end{aligned}
$$


where all maps are the natural projections and inclusions. The collection

$$
\mathfrak{q}=\left(\widehat{U}, u_{V}, z_{V}, P_{1}, \ldots, P_{2 \mu}\right)
$$

is a stable isometry structure of multiplicity $2 \mu$. By Theorem 1.7 there exists a $2 \mu$-component $r$-connected Seifert manifold $U=U_{1} \cup \cdots \cup U_{2 \mu}$ realizing $\mathfrak{q}$ (i.e., having $q$ as its stable isometry structure). Now, the union $U_{1} \cup \cdots \cup U_{\mu}$ is a $\mu$-component Seifert manifold whose stable isometry structure is isomorphic to $\mathfrak{q}_{V}$ and $U_{\mu+1} \cup \cdots \cup U_{2 \mu}$ has stable isometry structure isomorphic to $\mathfrak{q}_{W}$. Therefore, by Theorem 1.6, there exists an isotopy of $S^{n+2}$ taking $V$ onto $U_{1} \cup \cdots \cup U_{\mu}$ with respect to the orientations; similarly, there exists an isotopy of $S^{n+2}$ taking $W$ onto $U_{\mu+1} \cup \cdots \cup U_{2 \mu}$ with orientation reversed.

Join each $U_{i}$ with $U_{i+\mu}(i=1, \ldots, \mu)$ by a 1 -handle $H_{i}=D^{n} \times[0,1]$ with $H_{i} \cap U_{i}=H_{i} \cap \partial U_{i}=D^{n} \times 0, H_{i} \cap U_{i+\mu}=H_{i} \cap \partial U_{i+\mu}=D^{n} \times 1, H_{i} \cap H_{j}=\varnothing$ for $i \neq j$, and $H_{i} \cap V_{j}=\varnothing$ for $j \neq i, i+\mu$. We also assume that the orientations on $H_{i}$ induced by that of $U_{i}$ and $U_{i+\mu}$, agree. Denote $U_{i} \cup H_{i} \cup U_{i+\mu}$ by $S_{i}$.

The set $S_{1} \cup S_{2} \cup \cdots \cup S_{\mu}=S$ is a $\mu$-component Seifert manifold with the corresponding stable isometry structure

$$
\mathfrak{q}_{S}=\left(\widehat{U}, u_{V}, z_{U}, P_{1}+P_{\mu+1}, P_{2}+P_{\mu+2}, \ldots, P_{\mu}+P_{2 \mu}\right) .
$$

It follows from our construction and from Corollary 2.6 that $S$ is almost closed. This means that by a small isotopy we can translate $U_{\mu+1}, U_{\mu+2}, \ldots, U_{2 \mu}$ into new positions $U_{\mu+1}^{\prime}, \ldots, U_{2 \mu}^{\prime}$ such that

$$
U_{i} \cap U_{i+\mu}^{\prime}=\partial U_{i}=\partial U_{i+\mu}^{\prime}
$$

and

$$
U_{i} \cap U_{j}^{\prime}=\varnothing
$$

for $j \neq i+\mu$. Thus $U_{1} \cup \cdots \cup U_{\mu}$ and $U_{\mu+1}^{\prime} \cup \cdots \cup U_{2 \mu}^{\prime}$ are contiguous. This proves the theorem.

4.3. Two stable isometry structures $\mathfrak{q}_{0}$ and $\mathfrak{q}_{1}$ will be called R-equivalent if there exists a sequence $\mathfrak{p}_{0}, \mathfrak{p}_{1}, \ldots, \mathfrak{p}_{N}$ of stable isometry structures with $\mathfrak{p}_{0}=\mathfrak{q}_{0}$ and $\mathfrak{p}_{N}=\mathfrak{q}_{1}$, and for each $i \in\{0,1, \ldots, N-1\}$ either $\mathfrak{p}_{i}$ is contiguous to $\mathfrak{p}_{i+1}$ or $\mathfrak{p}_{i+1}$ is contiguous to $\mathfrak{p}_{i}$. $R$-equivalence is an equivalence relation.

From Theorems 1.6 and 4.2 we obtain

\subsection{Corollary. The map}

$$
\left\{\begin{array}{l}
\text { types of } \mathscr{F} \text {-links } \\
\text { of dimension } n \\
\text { and multiplicity } \mu
\end{array}\right\} \rightarrow\left\{\begin{array}{l}
\text { R-equivalence classes of stable isometry } \\
\text { structures of dimension } n \text { and multiplicity } \mu
\end{array}\right\}
$$

which sends a link type into the R-equivalence class of the stable isometry structure of an arbitrary Seifert manifold of the link, is correctly defined. This map is a bijection from the subset of stable $\mathscr{F}$-links onto the set of $R$-equivalence classes of stable isometry structures on $\left[\frac{n+1}{3}\right]$-connected complexes.

4.5. Now we consider simple odd-dimensional links. A simple link is an $\mathscr{F}$ link of dimension $(2 q-1)$, which is $(q-1)$-simple. We will assume that $q \geq 3$; in this case the link will be stable. 
Any simple link of dimension $2 q-1$ admits a $(q-1)$-connected Seifert manifold $V=V_{1} \cup \cdots \cup V_{\mu}$ (cf. [G]). From Poincare duality it follows that $\widehat{V}$ has the homotopy type of a wedge of spheres of dimension $q$, and so the homotopy type of $\widehat{V}$ is completely determined by the free abelian group $H_{q}(\widehat{V})$. Moreover, the $S$-maps $u, z, \pi_{1}, \ldots, \pi_{\mu}$ from the stable isometry structure of $\widehat{V}$ are completely determined by the induced maps on $H_{q}(\widehat{V})$; thus we come to the following known algebraic notion of isometry structure (cf. [K, Mio, Ko]):

4.6. An $\varepsilon$-symmetric $(\varepsilon= \pm 1)$ isometry structure of multiplicity $\mu$ is a tuple

$$
\left(M,\langle,\rangle, z, \pi_{1}, \ldots, \pi_{\mu}\right),
$$

where $M$ is a finitely generated free abelian group, $\langle\rangle:, M \otimes M \rightarrow \mathbb{Z}$ is an $\varepsilon$-symmetric bilinear form, and $z, \pi_{1}, \ldots, \pi_{\mu}: M \rightarrow M$ are endomorphisms of $M$, such that

(i) $\langle$,$\rangle is unimodular;$

(ii) $\langle z x, y\rangle=\langle x,(1-z) y\rangle \quad \forall x, y \in M$;

(iii) $\left\langle\pi_{i} x, \pi_{j} y\right\rangle=0$ for $i \neq j$,

(iv) $\pi_{i} \circ \pi_{j}=\delta_{i j} \pi_{j}$

(v) $\pi_{1}+\pi_{2}+\cdots+\pi_{\mu}=1_{M}$.

We will denote an isometry structure simply by $M$, and consider the other objects (the bilinear form and endomorphisms) to define additional structures on $M$.

Let $M_{1}$ and $M_{2}$ be two $\varepsilon$-symmetric isometry structures of multiplicity $\mu$. We will say that $M_{1}$ is contiguous to $M_{2}$ iff there are homomorphisms

$$
\varphi: M_{1} \rightarrow M_{2}, \quad \psi: M_{2} \rightarrow M_{1}
$$

such that for each $x \in\{1, \ldots, \mu\}, a \in M_{1}$, and $b \in M_{2}$ the following hold:

(a) $\langle a, \psi(b)\rangle=\langle\varphi(a), b\rangle$;

(b) $\varphi \pi_{k} z(a)=z \pi_{k} \varphi(a)$;

(c) $\psi \pi_{k} \bar{z}(b)=\bar{z} \pi_{k} \psi(b)$;

(d) $\varphi \pi_{k} \psi(b)=z \pi_{k} \bar{z}(b)$;

(e) $\psi \pi_{k} \varphi(a)=\bar{z} \pi_{k} z(a)$.

The equivalence relation generated by contiguity will be called $R$-equivalence.

Every Seifert manifold $V$ of a $(2 q-1)$-dimensional link determines an isometry structure (on the Betti group $H_{q}(\widehat{V}) /$ Tors) and contiguous Seifert manifolds determine contiguous isometry structures.

4.7. Theorem. For $q \geq 3$ the correspondence described above gives a bijection $\left\{\begin{array}{l}\text { types of simple } \\ \mathscr{F} \text {-links of dimension } \\ 2 q-1 \text { and multiplicity } \mu\end{array}\right\} \leftrightarrow\left\{\begin{array}{l}\text { R-equivalence classes of } \\ (-1)^{q} \text {-symmetric isometry } \\ \text { structures of multiplicity } \mu\end{array}\right\}$. Proof. The proof follows from Theorems 1.6, 2.6, and 4.2.

\section{HOMOLOGY PAIRINGS}

In this section we use Poincare duality to study the quadratic structure of the homology of the free covering of a boundary link. We construct two quadratic forms, which generalize well-known forms on the homology of infinite cyclic covering of knots: the Blanchfield form [B] and the torsion form [L4, F1].

Another generalization of the Blanchfield form was suggested by Duval [D]. 
5.1. Let $(\Sigma, \mathscr{S})$ be an $n$-dimensional $\mu$-component $\mathscr{F}$-link. Denote by $X$ the complement of an open tubular neighbourhood of $\Sigma$ in $S^{n+2}$. The splitting $\mathscr{S}$ determines a homomorphism of $\pi_{1}(X, *)$ onto $F_{\mu}$ (the free group in $t_{1}, \ldots, t_{\mu}$ ), which is defined up to conjugation. The covering $\widetilde{X} \rightarrow X$ corresponding to the kernel of the homomorphism $\pi_{1}(X, *) \rightarrow F_{\mu}$ is called the free covering, and has the free group $F_{\mu}$ as the group of covering translations. Homology groups $H_{*}(\tilde{X} ; \mathbb{Z})$, considered as left modules over $\Lambda=\mathbb{Z}\left[F_{\mu}\right]$, are invariants of $(\Sigma, \mathscr{S})$.

The modules $H_{*}(\tilde{X} ; \mathbb{Z})$ possess finite presentations (since $\Lambda$ is coherent) and are of type $L$ (cf. [S]): a module $M$ over $\Lambda$ is of type $L$ if the map

$$
\bigoplus_{i=1}^{\mu} M \rightarrow M, \quad\left(m_{1}, \ldots, m_{\mu}\right) \mapsto \sum_{i=1}^{\mu}\left(t_{i}-1\right) m_{i},
$$

where $m_{i} \in M, i=1, \ldots, \mu$, is a $\mathbb{Z}$-isomorphism.

5.2. Fix some notation: Let ${ }^{-}: \Lambda \rightarrow \Lambda$ denote the anti-isomorphism defined by

$$
\bar{t}_{i}=t_{i}^{-1}, \quad i=1, \ldots, \mu .
$$

If $M$ is a left $\Lambda$-module, $\bar{M}$ denotes the right $\Lambda$-module structure on $M$ given by

$$
m \lambda=\bar{\lambda} m, \quad m \in M, \lambda \in \Lambda .
$$

Let $e^{i}(M)$ denote $\operatorname{Ext}_{\Lambda}^{i}(M ; \Lambda)$-the right $\Lambda$-module. Let $\tau M$ denote the $\mathbb{Z}$-torsion part of $M, \tau M=\operatorname{Tors}_{\mathbb{Z}} M$, and let $f M=M / \tau M$ denote the $\mathbb{Z}$ torsion-free part of $M$.

5.3. Lemma [D]. Let $M$ be a finitely presented module of type $L$. Then

(1) $e^{0}(M)=0$;

(2) $e^{1}(M)$ has no $\mathbb{Z}$-torsion;

(3) $e^{1}(M) \approx e^{1}(f M)$;

(4) $e^{2}(M)$ is $\mathbb{Z}$-torsion;

(5) $e^{2}(M) \approx e^{2}(\tau M)$.

In the next two lemmas we shall compute $e^{1}$ and $e^{2}$. Let $I$ denote the augmentation ideal of $\Lambda$.

5.4. Lemma. Let $M$ be a finitely presented $\Lambda$-module of type $L$ and let $N$ be an arbitrary left $\Lambda$-module.

(1) If $\bigcap_{n=0}^{\infty} I^{n} N=0$, then $\operatorname{Hom}_{\Lambda}(M, N)=0$.

(2) If $\bigcap_{n=0}^{\infty} I^{n} N=0$ and $N$ is complete in the I-adic topology, then

$$
\operatorname{Ext}_{\Lambda}^{1}(M, N)=0 .
$$

Proof. The first statement is evident since $I^{n} M=M$ for any $n$.

To prove the second statement we have to show that any extension

$$
0 \rightarrow N \stackrel{\alpha}{\rightarrow} X \stackrel{\beta}{\rightarrow} M \rightarrow 0
$$

splits. We will do this by constructing a sequence of $\Lambda$-homomorphisms

$$
f_{n}: X \rightarrow N / I^{n} N, \quad n=0,1, \ldots,
$$


with the following properties:

(a) the diagram

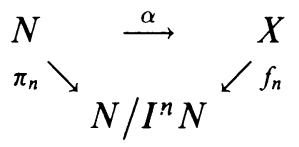

commutes (where $\pi_{n}$ is the projection);

(b) $f_{n+1}$ is a refinement of $f_{n}$ :

$$
N / I^{n} N \stackrel{f_{n}}{ } \longleftarrow \searrow^{f_{n+1}}
$$

By property (b) the sequence $\left\{f_{n}\right\}$ converges to a homomorphism $f: X \rightarrow N$ and by (a) this $f$ will be a splitting of the extension. Thus, the existence of such $\left\{f_{n}\right\}$ will prove $(2)$.

We will construct $f_{0}, f_{1}, \ldots$ inductively.

Put $f_{0}=0$ and suppose we have constructed $f_{0}, f_{1}, \ldots, f_{n}$ with the desired properties. To define $f_{n+1}$, consider arbitrary $x \in X$; then $\beta(x) \in M$ can be uniquely represented in the form

$$
\beta(x)=\sum_{i=1}^{\mu}\left(t_{i}-1\right) m_{i},
$$

where $m_{i} \in M, i=1, \ldots, \mu$. Now, $m_{i}=\beta\left(x_{i}\right)$ for some $x_{i} \in X$ and

$$
x=\sum_{i=1}^{\mu}\left(t_{i}-1\right) x_{i}+\alpha(\gamma),
$$

where $\gamma \in N$. Let us define $f_{n+1}(x)$ by

$$
f_{n+1}(x)=\sum_{i=1}^{\mu}\left(t_{i}-1\right) f_{n}\left(x_{i}\right)+\gamma \quad\left(\bmod I^{n+1} N\right) .
$$

If we choose other elements $x_{i}^{\prime} \in X$ with $\beta\left(x_{i}^{\prime}\right)=m_{i}$, then

$$
x_{i}^{\prime}=x_{i}+\alpha\left(\delta_{i}\right), \quad \delta_{i} \in N, i=1, \ldots, \mu,
$$

and we will have another representation

$$
x=\sum_{i=1}^{\mu}\left(t_{i}-1\right) x_{i}^{\prime}+\alpha\left(\gamma^{\prime}\right)
$$

with $\gamma^{\prime}=\gamma-\sum_{i=1}^{\mu}\left(t_{i}-1\right) \delta_{i}$. Hence, the result of computation of $f_{n+1}(x)$ according to the second formula will be

$$
\begin{aligned}
\gamma^{\prime}+ & \sum_{i=1}^{\mu}\left(t_{i}-1\right) f_{n}\left(x_{i}^{\prime}\right) \\
& =\gamma-\sum_{i=1}^{\mu}\left(t_{i}-1\right) \delta_{i}+\sum_{i=1}^{\mu}\left(t_{i}-1\right)\left[f_{n}\left(x_{i}\right)+f_{n}\left(\alpha\left(\delta_{i}\right)\right)\right] \\
& =\gamma+\sum_{i=1}^{\mu}\left(t_{i}-1\right) f_{n}\left(x_{i}\right) \quad\left(\bmod I^{n+1} N\right)
\end{aligned}
$$

and, thus, $f_{n+1}$ is correctly defined. 
It can be easily checked that $f_{n+1}$ is a $\Lambda$-homomorphism and

$$
f_{n+1}(x)=f_{n}(x) \quad\left(\bmod I^{n} N\right), \quad f_{n+1}(\alpha(\gamma))=\gamma \quad\left(\bmod I^{n+1} N\right),
$$

where $x \in X, \gamma \in N$. This proves the lemma.

Let $\Gamma$ be the ring of formal power series $\mathbb{Z}\left[\left[x_{1}, \ldots, x_{\mu}\right]\right]$ of noncommuting variables $x_{1}, \ldots, x_{\mu}$. We imbed $\Lambda$ in $\Gamma$ by the Magnus imbedding:

$$
t_{i} \rightarrow 1+x_{i}, \quad t_{i}^{-1} \rightarrow 1-x_{i}+x_{i}^{2}-x_{i}^{3}+\cdots .
$$

Let $\mathbb{Q} \Gamma$ be $\mathbb{Q}\left[\left[x_{1}, \ldots, x_{\mu}\right]\right], \mathbb{Q} \Lambda=\mathbb{Q}\left[F_{\mu}\right]$, and $\mathbb{Q} \Lambda \rightarrow \mathbb{Q} \Gamma$ be the similar imbedding.

5.5. Proposition. If $M$ is a finitely presented module of type $L$, then

(1) $e^{1}(M) \approx \operatorname{Hom}_{\Lambda}(M ; \Gamma / \Lambda)$;

(2) $e^{2}(M) \approx \operatorname{Hom}_{\Lambda}(\tau M ; \mathbb{Q} \Gamma /(\Gamma+\mathbb{Q} \Lambda))$,

where the sign $\approx$ means "naturally isomorphic as right $\Lambda$-modules."

Proof. (1) Lemma 5.4 says that

$$
\operatorname{Hom}_{\Lambda}(M ; \Gamma)=0=\operatorname{Ext}_{\Lambda}^{1}(M, \Gamma),
$$

so the exact sequence

$$
0=\operatorname{Hom}_{\Lambda}(M ; \Gamma) \rightarrow \operatorname{Hom}_{\Lambda}(M ; \Gamma / \Lambda) \rightarrow e^{1}(M) \rightarrow \operatorname{Ext}_{\Lambda}^{1}(M ; \Gamma)=0,
$$

derived from $0 \rightarrow \Lambda \rightarrow \Gamma \rightarrow \Gamma / \Lambda \rightarrow 0$, gives the isomorphism (1).

(2) By Lemma 5.3 we can assume that $M$ is $\mathbb{Z}$-torsion, $M=\tau M$. The exact sequence

$$
\operatorname{Ext}_{\Lambda}^{1}(M ; \mathbb{Q} \Lambda) \rightarrow \operatorname{Ext}_{\Lambda}^{1}(M ; \mathbb{Q} \Lambda / \Lambda) \rightarrow e^{2}(M) \rightarrow \operatorname{Ext}_{\Lambda}^{2}(M ; \mathbb{Q} \Lambda),
$$

derived from $0 \rightarrow \Lambda \rightarrow \mathbb{Q} \Lambda \rightarrow \mathbb{Q} \Lambda / \Lambda \rightarrow 0$, gives an isomorphism

$$
e^{2}(M) \approx \operatorname{Ext}_{\Lambda}^{1}(M ; \mathbb{Q} \Lambda / \Lambda),
$$

since $\operatorname{Ext}_{\Lambda}^{*}(M ; \mathbb{Q} \Lambda)=0(M$ is $\mathbb{Z}$-torsion $)$.

From Lemma 5.4 it follows that $\operatorname{Hom}_{\Lambda}(M ; \mathbb{Q} \Gamma / \Gamma)=0=\operatorname{Ext}_{\Lambda}^{1}(M ; \mathbb{Q} \Gamma / \Gamma)$. The exact sequence

$$
\begin{gathered}
\operatorname{Hom}_{\Lambda}(M ; \mathbb{Q} \Gamma / \Gamma) \rightarrow \operatorname{Hom}_{\Lambda}(M ; \mathbb{Q} \Gamma /(\Gamma+\mathbb{Q} \Lambda)) \\
\rightarrow \operatorname{Ext}_{\Lambda}^{1}(M ; \mathbb{Q} \Lambda / \Lambda) \rightarrow \operatorname{Ext}_{\Lambda}^{1}(M ; \mathbb{Q} \Gamma / \Gamma),
\end{gathered}
$$

derived from $0 \rightarrow \mathbb{Q} \Lambda / \Lambda \rightarrow \mathbb{Q} \Gamma / \Gamma \rightarrow \mathbb{Q} \Gamma /(\Gamma+\mathbb{Q} / \Lambda) \rightarrow 0$, gives

$$
\operatorname{Ext}_{\Lambda}^{1}(M ; \mathbb{Q} \Lambda / \Lambda) \approx \operatorname{Hom}_{\Lambda}(M ; \mathbb{Q} \Gamma /(\Gamma+\mathbb{Q} \Lambda)),
$$

which proves the lemma.

5.6. Let $(\Sigma, \mathscr{S})$ be an $n$-dimensional $\mu$-component $\mathscr{F}$-link and let $\tilde{X} \rightarrow$ $X$ be the corresponding free covering. Consider the chain complex $C$ of $\tilde{X}$ constructed by a cell-decomposition of $X$. The universal coefficient spectral sequence $[E C]$ gives an exact sequence

$$
0 \rightarrow e^{2}\left(H_{i-2}(C)\right) \rightarrow H^{i}(C ; \Lambda) \rightarrow e^{1}\left(H_{i-1}(C)\right) \rightarrow 0 .
$$


According to Lemma 5.3, $e^{2}\left(H_{i-2}(C)\right)$ coincides with the $\mathbb{Z}$-torsion subgroup of $H^{i}(C ; \Lambda)$.

The Poincare duality isomorphism [M1]

$$
H_{n+2-i}(\tilde{X}, \partial \tilde{X}) \rightarrow \overline{H^{i}(C, \Lambda)}
$$

gives two families of isomorphisms

$$
\begin{gathered}
\tau H_{n+2-i}(\tilde{X}, \partial \tilde{X}) \rightarrow \overline{e^{2}\left(H_{i-2}(C)\right)}, \\
B_{n+2-i}(\tilde{X}, \partial \widetilde{X}) \rightarrow \overline{e^{1}\left(H_{i-1}(C)\right)},
\end{gathered}
$$

where $B_{j}(Y)=f H_{j}(Y ; \mathbb{Z})$ denotes the Betti groups. It is clear that $\tau H_{j}(\tilde{X}, \partial \tilde{X})$ $=\tau H_{j}(\widetilde{X})$ for all $j$ and $B_{j}(\widetilde{X}, \partial \widetilde{X})=B_{j}(\widetilde{X})$ for $1 \leq j \leq n$. These identifications and Proposition 5.5 allow us to rewrite as

$$
\begin{aligned}
T_{j}(\tilde{X}) & \approx \overline{\operatorname{Hom}_{\Lambda}\left(T_{n-j}(\tilde{X}) ; \mathbb{Q} \Gamma /(\Gamma+\mathbb{Q} \Lambda)\right)}, \\
B_{j}(\tilde{X}) & \approx \overline{\operatorname{Hom}_{\Lambda}\left(B_{n+1-j}(\tilde{X}) ; \Gamma / \Lambda\right)}, \quad 1 \leq j \leq n,
\end{aligned}
$$

where $T_{j}$ denotes $\tau H_{j}$. From this we obtain the following theorem.

5.7. Theorem. In the notation of $\S 5.6$, there exist two families of forms:

$$
\begin{gathered}
{[,]: T_{i}(\tilde{X}) \times T_{n-i}(\tilde{X}) \rightarrow \mathbb{Q} \Gamma /(\Gamma+\mathbb{Q} \Lambda),} \\
\langle,\rangle: B_{i}(\tilde{X}) \times B_{n+1-i}(\tilde{X}) \rightarrow \Gamma / \Lambda, \quad 1 \leq i \leq n .
\end{gathered}
$$

These forms are conjugate-linear and nonsingular.

In the special case $\mu=1$ (knots) the pairing $\langle$,$\rangle was constructed by$ Blanchfield [B], and the pairing [, ] by Levine [L4] and the author [F1]. Another version of $\langle$,$\rangle was constructed by Duval [D].$

5.8. In order to obtain explicit computational formulas for $\langle$,$\rangle and [, ], we$ have to find the homomorphisms

$$
\begin{aligned}
\kappa: H^{i}(C ; \Lambda) & \rightarrow \operatorname{Hom}_{\Lambda}\left(H_{i-1}(C) ; \Gamma / \Lambda\right), \\
\chi: \operatorname{Tors}_{\mathbb{Z}} H^{i}(C ; \Lambda) & \rightarrow \operatorname{Hom}_{\Lambda}\left(T_{i-2}(C) ; \mathbb{Q} \Gamma /(\Gamma+\mathbb{Q} \Lambda)\right)
\end{aligned}
$$

forming the exact sequence (1) in $\S 5.6$.

Let $f: C_{i} \rightarrow \Lambda$ be a cocycle. Since $f\left(Z_{i}\right)=0, f$ defines a homomorphism $f_{1}: B_{i-1} \rightarrow \Lambda$ with $f_{1} \circ \partial=f$. By Lemma $5.4 f_{1}$ can be extended to a homomorphism $f_{2}: Z_{i-1} \rightarrow \Gamma$ and this extension is unique. Now $f_{1}$ and $f_{2}$ give a homomorphism

$$
\varphi: Z_{i-1} / B_{i-1}=H_{i-1}(C) \longrightarrow \Gamma / \Lambda
$$

and hence we have a homomorphism

$$
\kappa: H^{i}(C ; \Lambda) \rightarrow \operatorname{Hom}\left(H_{i-1}(C) ; \Gamma / \Lambda\right), \quad \kappa([f])=\varphi .
$$

In order to see that $\kappa$ is an epimorphism, we have to show that every homomorphism $Z_{i-1} \rightarrow \Gamma / \Lambda$ can be lifted to a homomorphism $Z_{i-1} \rightarrow \Gamma$. The obstruction to this is an element of $e^{1}\left(Z_{i-1}\right) \approx e^{2}\left(B_{i-2}\right)=0$. 
Let us assume now that $[f] \in H^{i}(C ; \Lambda)$ is of finite order. Then $f_{2}$ assumes its values in $\Lambda$. We want to construct the following commutative diagram

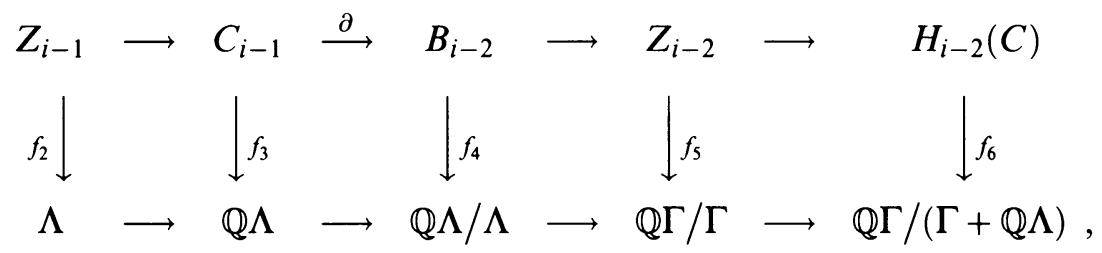

where all horizontal maps are "natural." The obstruction to constructing $f_{3}$ lies in $\operatorname{Ext}_{\Lambda}^{1}\left(B_{i-2}, \mathbb{Q} \Lambda\right)=\operatorname{Ext}_{\mathbb{Q} \Lambda}^{1}\left(\mathbb{Q} \otimes_{\mathbb{Z}} B_{i-2} ; \mathbb{Q} \Lambda\right)$ and the last group is trivial since $\mathbb{Q} \otimes_{\mathbb{Z}} B_{i-2}$ is a submodule of a free $\mathbb{Q} \Lambda$-module $\mathbb{Q} \otimes_{\mathbb{Z}} C_{i-2}$; so the result now follows from the fact that $\mathbb{Q} \Lambda$ is a free-ideal ring [C]. $f_{3}$ obviously defines $f_{4}$. The existence of $f_{5}$ is guaranteed by Lemma 5.4 , and $f_{6}$ is determined obviously. We have an ambiguity in the construction of $f_{3}$ and $f_{5}$. It is clear that $f_{6}$ is correctly defined modulo $\beta_{*} \operatorname{Hom}\left(H_{i-2}(C) ; \mathbb{Q} \Gamma / \mathbb{Q} \Lambda\right)$, where $\beta: \mathbb{Q} \Gamma / \mathbb{Q} \Lambda \rightarrow \mathbb{Q} \Gamma /(\Gamma+\mathbb{Q} \Lambda)$ is the projection. We will show later that

$$
\begin{aligned}
\sigma: \operatorname{Hom}\left(H_{i-2}(C) ; \mathbb{Q} \Gamma /(\Gamma+\mathbb{Q} \Lambda)\right) / \beta_{*} \operatorname{Hom}\left(H_{i-2}(C) ; \mathbb{Q} \Gamma / \mathbb{Q} \Lambda\right) \\
\quad \rightarrow \operatorname{Hom}\left(\tau H_{i-2}(C) ; \mathbb{Q} \Gamma /(\Gamma+\mathbb{Q} \Lambda)\right),
\end{aligned}
$$

which is given by taking the restriction to $\tau H_{i-2}(C)$, is an isomorphism; and (7) any homomorphism $Z_{i-2} \rightarrow \mathbb{Q} \Gamma /(\Gamma+\mathbb{Q} \Lambda)$ can be lifted to $Z_{i-2} \rightarrow \mathbb{Q} \Gamma / \Gamma$. Assuming that (6), (7) are true, we see that the above arguments give the map

$$
\begin{gathered}
\kappa: \tau H^{i}(C ; \Lambda) \rightarrow \operatorname{Hom}\left(\tau H_{i-2}(C) ; \mathbb{Q} \Gamma /(\Gamma+\mathbb{Q} \Lambda)\right), \\
\chi([f])=\left.f_{6}\right|_{\tau H_{i-2}(C)} .
\end{gathered}
$$

The fact that $\chi$ is an epimorphism follows immediately from (6) and (7). If $\chi[f]=0$, then we can construct a diagram as above with $f_{6}=0$; hence $f_{3}$ assumes its values in $\Lambda$ and is a cochain with $\delta f_{3}=f$. Thus $\chi$ is an isomorphism.

Let us now prove (7). By virtue of the exact sequence

$$
0 \rightarrow \mathbb{Q} \Lambda / \Lambda \rightarrow \mathbb{Q} \Gamma / \Gamma \rightarrow \mathbb{Q} \Gamma /(\Gamma+\mathbb{Q} \Lambda) \rightarrow 0
$$

the obstruction to lifting $Z_{i-2} \rightarrow \mathbb{Q} \Gamma /(\Gamma+\mathbb{Q} \Lambda)$ lies in $\operatorname{Ext}_{\Lambda}^{1}\left(Z_{i-2}, \mathbb{Q} \Lambda / \Lambda\right)$ which is trivial.

Statement (6) follows from the following lemma.

5.9. Lemma. Let $M$ be a finitely generated $\Lambda$-module of type $L$. Then

(a) any homomorphism $\tau M \rightarrow \mathbb{Q} \Gamma /(\Gamma+\mathbb{Q} \Lambda)$ can be extended to $M \rightarrow$ $\mathbb{Q} \Gamma /(\Gamma+\mathbb{Q} \Lambda)$

(b) if $\tau M=0$ then any homomorphism $M \rightarrow \mathbb{Q} \Gamma /(\Gamma+\mathbb{Q} \Lambda)$ can be lifted to $\mathbb{Q} \Gamma / \mathbb{Q} \Lambda$.

Proof. (a) Let $f M=M / \tau M$. The obstruction to constructing the extension lies in $\operatorname{Ext}_{\Lambda}^{1}(f M ; \mathbb{Q} \Gamma /(\Gamma+\mathbb{Q} \Lambda))$. The exact sequence

$$
0 \rightarrow \mathbb{Q} \Lambda / \Lambda \rightarrow \mathbb{Q} \Gamma / \Gamma \rightarrow \mathbb{Q} \Gamma /(\Gamma+\mathbb{Q} \Lambda) \rightarrow 0
$$


gives an exact sequence

$$
\operatorname{Ext}_{\Lambda}^{1}(f M ; \mathbb{Q} \Gamma / \Gamma) \rightarrow \operatorname{Ext}_{\Lambda}^{1}(f M ; \mathbb{Q} \Gamma /(\Gamma+\mathbb{Q} \Lambda)) \rightarrow \operatorname{Ext}_{\Lambda}^{2}(f M ; \mathbb{Q} \Lambda / \Lambda),
$$

where both side groups are zero (by virtue of Lemma 5.4 and Proposition 4.1 of [D]).

Let us now prove (b). The obstruction to constructing the lifting lies in $\operatorname{Ext}_{\Lambda}^{1}(M ; \Gamma / \Lambda)$. The exact sequence $0 \rightarrow \Lambda \rightarrow \Gamma \rightarrow \Gamma / \Lambda \rightarrow 0$ gives an exact sequence

$$
\operatorname{Ext}_{\Lambda}^{1}(M ; \Gamma) \rightarrow \operatorname{Ext}_{\Lambda}^{1}(M ; \Gamma / \Lambda) \rightarrow e^{2}(M),
$$

where both side groups are zero (by Lemmas 5.3 and 5.4).

The above arguments give the following recipes for calculation of $\kappa$ and $\chi$ in $\S 5.8$ :

5.10. Proposition. Let $f: C_{i} \rightarrow \Lambda$ be a cocycle. Then the homomorphism $\kappa[f]: H_{i-1}(C) \rightarrow \Gamma / \Lambda$ acts as follows: For any cycle $c \in Z_{i-1}$ there is a sequence of $i$-dimensional chains $\alpha_{m}$ with $\partial a_{m}$ converging to $c$ in the I-adic topology, and

$$
\kappa([f])([c])=\lim _{m \rightarrow \infty} f\left(\alpha_{m}\right) \bmod \Lambda .
$$

Here $f\left(a_{m}\right) \in \Lambda$ and the limit is an element of $\Gamma$. Its class modulo $\Lambda$ does not depend on the sequence $\left\{\alpha_{m}\right\}$.

5.11. Proposition. Assume that $f: C_{i} \rightarrow \Lambda$ is a cocyle representing a $\mathbb{Z}$-torsion cohomology class. It defines a homomorphism

$$
\chi[f]: \tau H_{i-2}(C) \rightarrow \mathbb{Q} \Gamma /(\Gamma+\mathbb{Q} \Lambda)
$$

acting as follows. Let $c \in Z_{i-2}$ represent a $\mathbb{Z}$-torsion homology class. There exist sequences $\alpha_{m} \in C_{i-1}$ and $\beta_{m} \in C_{i}$, and elements $\gamma \in C_{i-1}$ and $N \in \mathbb{Z}$, $N \neq 0$, such that
(a) $N c=\partial \gamma$;
(b) $\partial \alpha_{m} \rightarrow c$ in $C_{i-2}$; and
(c) $N \alpha_{m}-\partial \beta_{m}-\gamma \rightarrow 0$ in $C_{i-1}$.

Then

$$
\chi([f])([c])=\frac{1}{N} \lim _{m \rightarrow \infty} f\left(\beta_{m}\right),
$$

where the limit is taken in $\mathbb{Q} \Gamma$ and should be considered modulo $\Gamma+\mathbb{Q} \Lambda$. The result does not depend on the choice.

\section{REFERENCES}

[B] R. C. Blanchfield, Intersection theory of manifolds with operators with applications to knot theory, Ann. of Math. (2) 65 (1957), 340-356.

[CS] S. E. Cappell and J. L. Shaneson, Link cobordism, Comment. Math. Helv. 55 (1980), 29-49.

[C] P. M. Cohn, Free rings and their relations, Academic Press, London, New York, 1971.

[D] J. Duval, Forme de Blanchfield et cobordisme d'entrelacs bords, Comment. Math. Helv. 61 (1986), 617-635.

[EC] S. Eilenberg and H. Cartan, Homological algebra, Princeton Univ. Press, Princeton, N.J., 1956.

[F1] M. Farber, Duality in an infinite cyclic covering and even-dimensional knots, Math. USSR-Izv. 11 (1977), no 4, 749-781. 


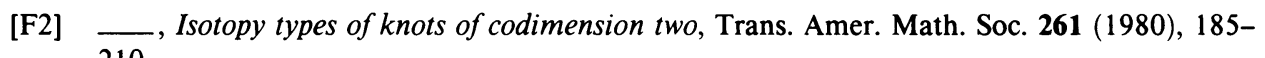
210.

[F3] _ Classification of simple knots, Russian Math. Surveys 38 (1983), 63-117.

[F4] An algebraic classification of some even-dimensional spherical knots. I, II, Trans. Amer. Math. Soc. 281 (1984), 507-527, 529-570.

[F5] _-, Hermitian forms on link modules, Comment. Math. Helv. 66 (1991), 189-236.

[Fr] P. Freyd, Splitting homotopy idempotents, Proc. Conf. on Categorical Algebra (La Jolla, 1965), Springer-Verlag, Berlin-Heidelberg-New York, 1966.

[G] M. A. Gutiérrez, Boundary links and an unlinking theorem, Trans. Amer. Math. Soc. 171 (1972), 491-499.

[K1] C. Kearton, Classification of simple knots by Blanchfield duality, Bull. Amer. Math. Soc. 79 (1973), 952-955.

[K2] _ B Blanchfield duality and simple knots, Trans. Amer. Math. Soc. 202 (1975), 141-160.

[KL] C. Kearton and W. B. R. Lickorish, Piecewise linear critical levels and collapsing, Trans. Amer. Math. Soc. 170 (1972), 415-424.

[K] M. A. Kervaire, Knot cobordism in codimension two, Lecture Notes in Math., vol. 197, Springer-Verlag, 1970, pp. 83-105.

[Ko] K. H. Ko, Seifert matrices and boundary link cobordisms, Trans. Amer. Math. Soc. 299 (1987), 657-681.

[Ko1] _ _ Seifert matrices and boundary links, Ph.D. thesis, Brandeis University, 1984.

[L1] J. Levine, Polynomial invariants of knots of codimension two, Ann. of Math. (2) 84 (1966), 537-554.

[L2] _ Knot cobordism group in codimension two, Comment. Math. Helv. 44 (1969), 229-244.

[L3] _ An algebaic classification of some knots of codimension two, Comment. Math. Helv. 45 185-198 (1970).

[L4] _ Knot modules. I, Trans. Amer. Math. Soc. 229 (1977), 1-50.

[L] C. Liang, An algebraic classification of some links of codimension two, Proc. Amer. Math. Soc. 67 (1977), 147-151.

[M1] J. Milnor, A duality theorem for Reidemeister torsion, Ann. of Math. (2) 76 (1962), 137-147.

[M2] _ Infinite cyclic coverings, Conference on the Topology of Manifolds, Prindle, Weber \& Schmidt, Boston, Mass., 1968, pp. 115-133.

[Mio] W. Mio, On boundary link cobordism, Math. Proc. Cambridge Philos. Soc. 101 (1987), 259266.

[Tr] H. F. Trotter, On S-equivalence of Seifert matrices, Invent. Math. 20 (1973), 173-207.

[S] N. Sato, Free coverings and modules of boundary links, Trans. Amer. Math. Soc. 264 (1981), 499-505.

[Sm] N. Smythe, Boundary links, Wisconsin Topology Seminar, Ann. of Math. Studies, no. 60, Princeton Univ. Press, Princeton, N.J., 1965.

[St] R. E. Stong, Notes on cobordism theory, Princeton Univ. Press, 1968.

[W] C. T. C. Wall, Classification problems in differential topology. IV, Thickenings, Topology 5 (1966), 73-94.

Department of Mathematics, Raymond and Beverly Sackler Faculty of Exact Sciences, Tel Aviv University, Ramat-Aviv, Tel Aviv 69978, IsRael

E-mail address: Farber@math.tau.ac.il 